\title{
Audit of vitamin D intake in a nursing home (NH) for the elderly mentally infirm (EMI)
}

\author{
W. Milligan ${ }^{1}$, C. Bridges ${ }^{2}$ and T. Christides ${ }^{2}$ \\ ${ }^{1}$ Department of Nutrition \& Dietetics, Medway Community Healthcare, St Bartholomew's Hospital, Rochester, ME1 1DS \\ and ${ }^{2}$ University of Greenwich at Medway, Chatham Maritime, ME4 4TB, UK
}

Vitamin D is essential for musculoskeletal health. Deficiency results in impaired absorption of dietary calcium, and in adults may cause osteomalacia $^{(1)}$. Elderly NH residents are at high-risk of vitamin D deficiency, due to reduced sunlight exposure, and physiological changes of ageing resulting in decreased photosynthetic production of vitamin $\mathrm{D}^{(1)}$. The Department of Health (DH) recommends a vitamin D supplement of $10 \mu \mathrm{g} / \mathrm{d}$ for institutionalised people and those aged 65 years and over ${ }^{(2)}$. Data on implementation of this recommendation are scant. The aim of this audit was to determine if DH recommendations for vitamin D intake were being met in a group of institutionalised EMI residents. The audit met the local ethics guidance.

Participants were residents of a NH for the EMI. 12 participants out of 40 residents $(30 \%)$ were randomly selected. Average age was 79.7 years (range 59-87 y). Vitamin D intake was estimated by household measures food diary technique; records were kept by nursing staff for seven days and analysed using Dietplan 6. Data on supplements, medication and sunlight exposure were also collected.

All participants had daily intakes of vitamin D below the RNI, and lower than reported for a similar population ${ }^{(3)}$. The main vitamin D food sources were fruit yogurt, mashed potato and sponge cake $(80 \%)$, in contrast with results from the 'National Diet and Nutrition Survey: People Aged 65 Years and Over' in which oily fish, fat spreads and cereals were the major sources $(73 \%)^{(3)}$.

Table 1. Comparison of Vitamin D intake data

\begin{tabular}{lcccc}
\hline & \multicolumn{3}{c}{ Vitamin D intake $(\mu \mathrm{g} /$ day $)$} \\
\cline { 2 - 5 } & Mean & SD & Median & Range/lower, upper 2.5 percentile \\
\hline Local data $_{\text {NDNS data }}{ }^{3}$ & 2.37 & 1.1 & 2.58 & $0.58-4.05$ \\
\hline
\end{tabular}

No surveyed participants were on vitamin D supplements; $75 \%$ received no direct sunlight exposure, with the others receiving less than two hours over the 7 day period (the study, however, was undertaken in January). $25 \%$ of residents were on modified texture diets, and $75 \%$ were taking at least one medication that is recognised to have a negative effect on vitamin D status such as carbamazepine.

Neither vitamin D supplement nor dietary intake recommendations of the UK DH were met for any participants. Low dietary intake may be secondary to special EMI diet needs, such as modified texture diets that limit the use of vitamin D-rich food sources. The finding of this audit suggest that the institutionalised EMI are not receiving recommended supplements, and may be at particularly high risk for vitamin D deficiency because of special dietary needs and medication use. Further research across institutions is needed to confirm these findings and identify barriers to the provision of vitamin D adequate diets and recommended supplements.

1. Scientific Advisory Committee on Nutrition. (2007) Update on Vitamin D. London, TSO.

2. Department of Health (1998) Nutrition and Bone Health: with particular reference to calcium and vitamin D no. 49. London: The Stationery Office.

3. Finch S, Doyle W, Lowe C et al. (1998) National Diet and Nutrition Survey: people aged 65 years and over. London: The Stationery Office. 